\title{
Complete Characterization of Functions Satisfying the Conditions of Arrow's Theorem
}

\author{
Elchanan Mossel* and Omer Tamuz ${ }^{\dagger}$
}

April 15, 2011

\begin{abstract}
Arrow's theorem implies that a social welfare function satisfying Transitivity, the Weak Pareto Principle (Unanimity) and Independence of Irrelevant Alternatives (IIA) must be dictatorial. When non-strict preferences are also allowed, a dictatorial social welfare function is defined as a function for which there exists a single voter whose strict preferences are followed. This definition allows for many different dictatorial functions, since non-strict preferences of the dictator are not necessarily followed. In particular, we construct examples of dictatorial functions which do not satisfy Transitivity and IIA. Thus Arrow's theorem, in the case of nonstrict preferences, does not provide a complete characterization of all social welfare functions satisfying Transitivity, the Weak Pareto Principle, and IIA.

The main results of this article provide such a characterization for Arrow's theorem, as well as for follow up results by Wilson. In particular, we strengthen Arrow's and Wilson's result by giving an exact if and only if condition for a function to satisfy Transitivity and IIA (and the Weak Pareto Principle). Additionally, we derive formulae for the number of functions satisfying these conditions.
\end{abstract}

\section{Introduction}

\subsection{Arrow's Model and Non-Strict Preferences}

Arrow's famous impossibility theorem [1,2] shows that a group of voters, each with a preference regarding a set of alternatives, has a limited number of ways to come to an agreement. In fact, under some restrictions, the only possible system is dictatorship, that is, the unqualified adoption of one particular voter's preference.

In Arrow's model, a set of voters each have a preference, or ranking, of a set of social alternatives. A Social Welfare Function (SWF) is a function from a set of voters' preferences to a single, "agreed" preference. The preferences (both of the voters and of the outcome) may not be strict, so two alternatives could be equally ranked. However, as may be expected, each preference relation is transitive in the usual sense that if alternative $x$ is preferable to $y$, and $y$ is preferable to $z$, then $x$ is preferable to $z$.

By Arrow's definition, there exists in a dictatorship a single voter whose preferences are always adopted by the SWF. However, the SWF is bound only to the dictator's strict preferences, so that

\footnotetext{
${ }^{*}$ Weizmann Institute and U.C. Berkeley. E-mail: mossel@stat.berkeley.edu. Supported by a Sloan fellowship in Mathematics, by BSF grant 2004105, by NSF Career Award (DMS 054829) by ONR award N00014-07-1-0506 and by ISF grant $1300 / 08$

${ }^{\dagger}$ Weizmann Institute. Supported by ISF grant 1300/08
} 
if the dictator decrees that $x$ is equivalent to $y$, then the SWF may choose any of the three possible relations between $x$ and $y$.

Arrow's theorem states that an SWF whose outcome is transitive, and which also satisfies IIA and the Weak Pareto Principle, must be dictatorial. Recall that Independence of Irrelevant Alternatives (IIA) requires that the relative ranking of two alternatives, as decided by the SWF, depend only on the relative rankings assigned to these alternatives by the voters. Hence, if the SWF, given a set of voters' preferences, prefers alternative $x$ to $y$, then it must also prefer $x$ to $y$ for an altered set of preferences, given that the relative rankings of $x$ and $y$ remains unchanged.

The second condition, the Weak Pareto Principle (WPP), asserts that when all voters have the same strict preference, then the SWF must concur and the agreed ranking equals this common ranking.

\subsection{What Happens when the Dictator Can't Decide?}

If one allows strict rankings only, then all dictatorial functions satisfy IIA and WPP, and so Arrow's theorem provides a complete "if and only if" characterization: an SWF satisfies IIA and WPP iff it is dictatorial. However, in the general case of non-strict preferences "If the dictator is indifferent between $\mathrm{x}$ and $\mathrm{y}$, presumably he will then leave the choice up to some or all of the other members of society," in the words of Arrow [1]. Hence, many different dictatorial functions are possible, and in particular ones that don't satisfy IIA (we provide examples in Sect. 3 below). We are interested in finding a full characterization of the SWFs satisfying Arrow's theorem.

\subsection{Wilson's Framework}

We approach this problem under the slightly more general framework of Wilson [9], who proves an extension of Arrow's theorem. Further weakening the Weak Pareto Principle, Wilson assumes "Citizens' Sovereignty": that there exists no pair of alternatives $x$ and $y$ for which the SWF always deems $x$ strictly preferable to $y$, regardless of the voters' preferences. Under this weaker restriction, Wilson shows, in his first theorem, that a dictatorship still arises, albeit with a slightly modified definition of a dictator. Under Wilson's conditions, there exists one voter whose preferences are either always followed verbatim or always followed in reverse.

In his second theorem, Wilson disposes with the second condition entirely, leaving IIA only. He characterizes SWFs which satisfy IIA, but, like Arrow, does not give a full "if and only if" condition. In particular, and again like Arrow, he does not elaborate on how an SWF may decide on pairs which a dictator deems equal.

\subsection{Full Characterization}

We complete these gaps and give a full characterization of SWFs that satisfy the IIA condition. We show that functions satisfying Arrow's conditions defer decision, on pairs which the dictator deems equivalent, to a particular class of functions which we dub "clerical dictatorial". These functions divide each set of equivalent alternatives to an ordered partition, let a dictator decide on each partition and recursively defer the dictators' undecided pairs to other "clerical dictatorial" functions. Eventually, undecided pairs which are otherwise ordered with respect to the other alternatives can be decided by any SWF on two alternatives.

We use our characterization to count the number of SWFs satisfying IIA, the number of SWFs satisfying IIA and the Weak Pareto Principle, and the number of SWFs satisfying Arrow's conditions, i.e., IIA and the Weak Pareto Principle. 
Our characterization results in detailed understanding of the SWFs satisfying Arrow's and Wilson's conditions. One could argue that all the functions satisfying these conditions are undesirable voting systems, as they are very far from any notion of egalitarianism. However, the richness of the class of such functions as displayed by their large number may be somewhat surprising.

\subsubsection{Hierarchal Organizations}

A context in which these non-egalitarian functions may be desirable, or even natural, is that of a hierarchal organization. Of course there is no reason to assume organizational decision making should be transitive (see, e.g., [4, 5]), let alone satisfy IIA. However, assuming that individuals can be rational then hierarchal organizations can make rational decisions; for example, a dictatorship is a simple hierarchal structure that trivially results in rational organizational decisions, assuming the dictator is rational. We show that whenever organizational decisions satisfy Arrow's conditions then the organization is necessarily hierarchal and characterize the possible structures of hierarchy. This is not offered as a practical application in the field of organization design, but rather as a (hopefully) illuminating point of view on our results.

We claim that given a set of alternatives to be ranked by members of an organization, an SWF which satisfies Arrow's conditions effectively determines a "chain of command" with respect to each pair of alternatives $x$ and $y$. Each member of the chain, starting with the first, can either make a decision (i.e., $x<y$ or $y<x$ ) or remain indifferent and pass the decision onto the next in line. A chain can end with any SWF over two alternatives (e.g., majority vote or a fixed order), as long as the position of the pair among the rest of the alternatives has already been fixed. To satisfy Arrow's theorem, these chains cannot be arbitrary but rather have rich structure. In particular, there must exist one individual (Arrow's dictator) that is the first in all chains.

For example, consider a company that must rank alternatives $\{x, y, z, w\}$. To satisfy Arrow's conditions, it must be that the company has a CEO that has the prerogative of deciding the ranking. However, if she is indifferent with respect to $x$ and $y$ then the decision could be deferred to some subaltern executive, and if he is also indifferent then $x$ is ranked above $y$. At the same time, it could also be that when the CEO is indifferent with regards to the ranking of $z$ and $w$ then a majority vote is taken among the rest of the employees of the company. This (still not fully specified) SWF meets all the conditions of Arrow's theorem: IIA, WPP, and transitivity.

The different "chains of command" for the different pairs cannot be constructed arbitrarily. For example, it cannot be the case that the CEO defers to three distinct individuals the rankings of the three pairs $(x, y),(y, z)$ and $(x, z)$, since the resulting relation may not be transitive. The description of the exact conditions of how these chains may be constructed is the main aim of this article.

\subsubsection{Quantitative Arrovian Theorems}

This complete characterization may also play a role in proving quantitative versions of Arrow's theorem. Arrow's theorem states that a non-dictatorial SWF cannot satisfy IIA and transitivity simultaneously. However, it is natural to ask if such an SWF can have a transitive outcome for a large proportion of the voting profiles. Likewise, a natural question is whether there exists a non-dictatorial SWF with transitive outcomes, such that the IIA condition is met for most voting profiles.

Negative answers to the questions above are given in $[6,7]$ where it is shown that if a transitive function satisfies the IIA property for a large proportion of the profiles then it necessarily agrees with a dictatorial function on a large proportion of the profiles. The same holds for an IIA function whose outcome is transitive for a high proportion of the profiles. 
In [6] a quantitative Arrow theorem is proved for strict preferences, and only for a restricted sub-class of balanced SWFs (these are functions where for each pair of alternatives exactly half of the voting profiles result in one alternative preferred over the other). The proof in [7], which gives a complete quantitative version of Arrow's theorem for strict preferences, uses a characterization of all the functions satisfying IIA (for strict preferences). Thus, it proves more generally that if a function is IIA and close to being transitive then it must agree on a high proportion of the voting profiles with some functions that is always IIA and transitive. Hence, it seems that a general framework which looks at all IIA and transitive functions is crucial for quantitative proofs of Arrow's theorem. We expect that the results proven here will play a role in deriving quantitative versions of Arrow's theorem, where non-strict preferences are allowed.

\section{Definitions}

Basic notation:

- $S$ is a finite set of social states (alternatives). In the context of this article the interesting regime is $S \geq 3$.

- $\Pi_{S}$ is the set of complete transitive binary relations (weak preferences) on $S$.

We depart here from standard notation: given a binary relation $R$, we express $(x, y) \in R$ as $x \leq_{R} y$, rather than the often used $x R y$. This gives us the convenience of writing $x<_{R} y$ and $x=R y$, the meaning of which is clear when $R$ is complete.

Recall that $R$ is a complete transitive binary relation if it satisfies the following conditions:

1. $\forall x, y \in S, x \leq_{R} y$ or $y \leq_{R} x$ or both. As a consequence, $\forall x \in S$ it holds that $x \leq_{R} x$.

2. $\forall x, y, z \in S$, if $x \leq_{R} y$ and $y \leq_{R} z$ then $x \leq_{R} z$.

- $V=\{1, \ldots, N\}$ is a finite set of voters. Although most concepts we discuss below (e.g., IIA, Weak Pareto Principle) are already non-trivial when $N=1$, our regime of interest is $N \geq 2$.

- The preference of $i \in V$ is $\mathbf{R}_{i} \in \Pi_{S}$

- The preferences of all $N$ voters $\mathbf{R}=\left(\mathbf{R}_{1}, \ldots, \mathbf{R}_{N}\right)$ is called a configuration. Since a configuration can also be viewed as a function from the voters to preferences, we denote the set of configurations by $\Pi_{S}^{V}$.

- 0 is the "null voter," who always deems all alternatives equal. We introduce the null voter to simplify some of the statements to follow.

- $f: \Pi_{S}^{V} \rightarrow \Pi_{S}$ is a social welfare function (SWF). Note that by this definition, all SWFs are transitive, in the sense that their image consists of transitive relations.

The central property of SWFs that concerns us is Independence of Irrelevant Alternatives. The following is a standard definition, introduced by Arrow [1]:

Definition 2.1. An $S W F$ satisfies Independence of Irrelevant Alternatives (IIA) if, for $a$ given pair $x, y \in S$, it depends only on the voters' preferences on that pair. Alternatively, let an $S W F f$ satisfy IIA. Then, if configurations $\mathbf{P} \in \Pi_{S}^{V}$ and $\mathbf{Q} \in \Pi_{S}^{V}$ agree on $\{x, y\}$, then $f(\mathbf{P})$ agrees with $f(\mathbf{Q})$ on $\{x, y\}$. 
Note that any function with only one or two social states trivially satisfies IIA.

The following definition is also standard.

Definition 2.2. An SWF satisfies the Weak Pareto Principle (WPP) if, whenever all the voters agree that $x<y$, then $f$ also agrees on that preference: Let $f$ be an SWF satisfying WPP. Then $\forall i \in V: x<_{R_{i}} y$ implies $x<_{f(\mathbf{R})} y$.

Following Wilson, we make the following definition which is a further weakening of the Weak Pareto Principle:

Definition 2.3. An SWF satisfies Citizens' Sovereignty (CS) if for each pair of alternatives $\{x, y\}$, there exists a configuration $\mathbf{P}$ such that $x \leq_{f(\mathbf{P})} y$ and there exists a configuration $\mathbf{Q}$ such that $y \leq f(\mathbf{Q}) x$.

Wilson also makes this definition.

Definition 2.4. An $S W F f$ is null if for every configuration $\mathbf{R}$ and pair of alternatives $\{x, y\}$, it holds that $x={ }_{f(\mathbf{R})} y$.

Another natural definition is that of a dictatorial function:

Definition 2.5. An $S W F f$ is dictatorial if there exists a dictator in $V$ whose preferences are $a$ prerequisite for $f$ (perhaps inversely):

$$
\begin{aligned}
& (\exists i \in V)(\forall x, y \in S)\left(\forall \mathbf{R} \in \Pi_{S}^{V}\right): x \leq_{f(\mathbf{R})} y \rightarrow x \leq_{R_{i}} y \\
& (\exists i \in V)(\forall x, y \in S)\left(\forall \mathbf{R} \in \Pi_{S}^{V}\right): x \leq_{f(\mathbf{R})} y \rightarrow y \leq_{R_{i}} x .
\end{aligned}
$$

Let an SWF $f: \Pi_{S}^{V} \rightarrow \Pi_{S}$ be dictatorial, and let $i$ be a dictator whose preferences are a prerequisite for $f$ (directly, rather than inversely). Then, repeating the above, it holds that for each $x, y$ and $\mathbf{R}$,

$$
x \leq_{f(\mathbf{R})} y \rightarrow x \leq_{R_{i}} y .
$$

Now, this is equivalent to:

$$
x<_{R_{i}} y \rightarrow x<_{f(\mathbf{R})} y,
$$

so that one could, alternatively, define a dictator as a voter to whose strict preferences $f$ conforms. The relations among members of each equivalence class of $R_{i}$ are then left to be otherwise determined.

This discussion motivates the following definition:

Definition 2.6. Let $f: \Pi_{S}^{V} \rightarrow \Pi_{S}$ be a dictatorial $S W F$ with dictator $i$ and $g: \Pi_{S}^{V \backslash\{i\}} \rightarrow \Pi_{S}$ to be an $S W F$, with $i$ removed from the set of voters.

Given a configuration $\mathbf{R} \in \Pi_{S}^{V}$, we will write $\mathbf{R}_{-i}$ for the element of $\Pi_{S}^{V \backslash\{i\}}$ which agrees with $\mathbf{R}_{-i}$ for all voters in $V \backslash\{i\}$.

We say that $f$ defers to $g$ over $i$ if, whenever $x={ }_{R_{i}} y, f(\mathbf{R})$ agrees with $g\left(\mathbf{R}_{-i}\right)$ over $\{x, y\}$ :

$$
(\forall x, y \in S)\left(\forall \mathbf{R} \in \Pi_{S}^{V}\right): x=_{R_{i}} y \rightarrow\left(x \leq_{f(\mathbf{R})} y \leftrightarrow x \leq_{g\left(\mathbf{R}_{-i}\right)} y\right)
$$

Intuitively, the phrase " $f$ defers to $g$ over $i$ " means that the function $g$ is used to rank any pair for which the dictator $i$ is undecided. It is easy to see that for any dictatorial $f$ with dictator $i$ there may only exist a single $g$ such that " $f$ defers to $g$ over $i$ ".

Let a "cleric" be characterized by some fixed opinion $C \in \Pi_{S}$, which is independent of all the voters' preferences. 
Definition 2.7. An $S W F f$ is clerical with cleric $C$ if $C$ 's (fixed) preferences are a prerequisite for $f$ :

$$
(\forall x, y \in S)\left(\forall \mathbf{R} \in \Pi_{S}^{V}\right): x \leq_{f(\mathbf{R})} y \rightarrow x \leq_{C} y .
$$

Alternatively, if $C$ is a cleric for $f$, then $C$ 's strict preferences are followed by $f$, for all configurations. Note that for $f$ clerical with cleric $C$, if $\{x, y\}$ are such that $x \leq_{f(\mathbf{R})} y$ for some configurations, and $y \leq_{f(\mathbf{R})} x$ for some configurations (so that there is no strict fixed preference between them), then $x={ }_{C} y$, and $x$ and $y$ belong to the same equivalence class of $C$. Note also that every SWF is clerical with the trivial cleric who deems all alternatives equal.

Let $A \subset S$ be a set of alternatives and $f$ an SWF. The function $f$ restricted to $A$, denoted $\left.f\right|_{A}$, is the function $\left.f\right|_{A}: \Pi_{A}^{V} \rightarrow \Pi_{A}$ defined as follows. Given $\mathbf{R}=\left(\mathbf{R}_{1}, \ldots, \mathbf{R}_{n}\right) \in \Pi_{A}^{V}$, let $\mathbf{R}^{\prime}=\left(\mathbf{R}_{1}^{\prime}, \ldots, \mathbf{R}_{n}^{\prime}\right) \in \Pi_{S}^{V}$ agree with them on all the pairwise preferences in $A$. Then for $x, y \in A$ we have $x \leq_{\left.f\right|_{A}(\mathbf{R})} y$ iff $x \leq_{f\left(\mathbf{R}^{\prime}\right)} y$. Note that if (but not only if) IIA holds then $\left.f\right|_{A}$ is well defined, i.e. it does not depend on the choice of $\mathbf{R}^{\prime}$. This point has been hitherto noted by Blau [3].

Definition 2.8. An $S W F f: \Pi_{S}^{V} \rightarrow \Pi_{S}$ is clerical-dictatorial if it is null, if $S$ is of size at most two, or if there exists a cleric $C$ such that all of the following hold:

1. $f$ is a clerical function with cleric $C$.

2. Let $A$ be any of $C$ 's equivalence classes.

(a) $\left.f\right|_{A}$ is well defined, and is either null, dictatorial, or of size 2 .

(b) If $\mathrm{f}_{A}$ is dictatorial then it defers to a clerical-dictatorial function.

\section{Our Theorem}

In terms of the definitions above, Arrow's theorem [1] is the following:

Theorem 3.1 (Arrow). Any SWF which satisfies IIA and WPP is dictatorial (with the dictator's decrees followed verbatim rather than in reverse).

Wilson's first theorem (theorem 3 in [9]) is the following:

Theorem 3.2 (Wilson). Any SWF which satisfies IIA and CS is either dictatorial or null.

Wilson's second theorem (theorem 5 in [9]) is the following:

Theorem 3.3 (Wilson). Any SWF which satisfies IIA is clerical with some cleric $C$, so that restricted to $C$ 's equivalence classes of size $>2$, it is either dictatorial or null.

Note that none of the above is a complete characterization of the functions satisfying the respective conditions.

Example 3.4. Consider the following function $f$ on three social alternatives, $x, y$ and $z$, with two voters. Let voter 1 be a dictator, so that his strict preferences are followed verbatim. When voter 1 deems all three alternatives equal, then

- If voter 2 also votes $x=y=z$ then $f$ outputs $x=y=z$.

- Otherwise (for example, when voter 2 votes $x<y=z$ ), $f$ outputs $x=y<z$.

This function, although it is dictatorial (and even satisfies WPP), clearly does not satisfy IIA. 
By the definitions above, any SWF is transitive, in the sense that the relations in its image are transitive. If one allows for non-transitive preferences in the image, then it possible to construct a dictatorial function that is not transitive.

Example 3.5. Let $f$ be an $S W F$ on three social alternative and two voters, with the first voter taking the rôle of dictator. Then $f$ follows voter 1's strict preferences. When 1 deems two alternatives equal, then $f$ does too, if voter 2 agrees. When voter 2 disagrees, then, depending on the two alternatives in questions, $f$ outputs $x<y$ if the two alternatives are $x, y, y<z$ if the two alternatives are $y, z$ and $z<x$ if the two alternatives are $x, z$. Thus, when voter 1 votes $x=y=z$ and voter 2 votes $x<y<z$, then $f$ outputs $x<y<z$, but also $z<x$, and hence is not transitive. Note that this function satisfies IIA and WPP.

We provide a complete characterization of SWFs satisfying the conditions in theorems of Wilson and of Arrow.

Our "if and only if" version of Arrow's theorem is:

Theorem 3.6. An SWF satisfies IIA and WPP if and only if is dictatorial (with the dictator's decrees followed verbatim rather than in reverse), and defers to a clerical-dictatorial function.

Our "if and only if" version of Wilson's first theorem is:

Theorem 3.7. An SWF satisfies IIA and CS if and only if it is null or dictatorial, and in the latter case defers to a clerical-dictatorial function.

Our "if and only if" version of Wilson's second theorem is:

Theorem 3.8. An SWF satisfies IIA if and only if it is clerical-dictatorial.

We prove (3.8) below, and show that theorems (3.6) and (3.7) follow as corollaries.

\section{$3.1 \quad$ IIA $\Longrightarrow$ Clerical-Dictatorial}

In this section we prove the following theorem:

Theorem 3.9. Any SWF which satisfies IIA is clerical-dictatorial.

A key concept in the proof will be the relation $C(f)$, defined as follows: $C(f)$ is the relation signifying that, between social states $x$ and $y$, for some configuration, $x \leq_{f} y$. Formally,

$$
x \leq_{C(f)} y \quad \leftrightarrow \quad \exists \mathbf{R} \in \Pi_{S}^{V}: x \leq_{f(\mathbf{R})} y
$$

Note that $x<_{C(f)} y$ iff for all configurations, $x<_{f(\mathbf{R})} y$.

We will use the following lemma which is essentially due to Wilson.

Lemma 3.10. $C(f)$ is complete and transitive.

Proof. Completeness: let $\mathbf{R}$ be some configuration. Then either $x \leq_{f(\mathbf{R})} y$ or $y \leq_{f(\mathbf{R})} x$. Hence $x \leq_{C(f)} y$ or $y \leq_{C(f)} x$, and $C(f)$ is complete.

Transitivity (see Fig. 3.1): let $x \leq_{C(f)} y$ and $y \leq_{C(f)} z$. Then there exist configurations $\mathbf{P}$ and $\mathbf{Q}$ s.t. $x \leq_{f(\mathbf{P})} y$ and $y \leq_{f(\mathbf{Q})} z$. Let $\mathbf{R}$ be a configuration that agrees with $\mathbf{P}$ on $\{x, y\}$ and with $\mathbf{Q}$ on $\{y, z\}$. Such a configuration always exists, since preferences on $\{x, y\}$ and $\{y, z\}$ can always be completed to a transitive relation by some ordering of $\{x, z\}$.

Because of IIA, $x \leq_{f(\mathbf{R})} y$ and $y \leq_{f(\mathbf{R})} z$. Finally, because $f(\mathbf{R})$ is transitive, it follows that $x \leq_{f(\mathbf{R})} z$, and hence, by the definition of $C(f), x \leq_{C(f)} z$. 


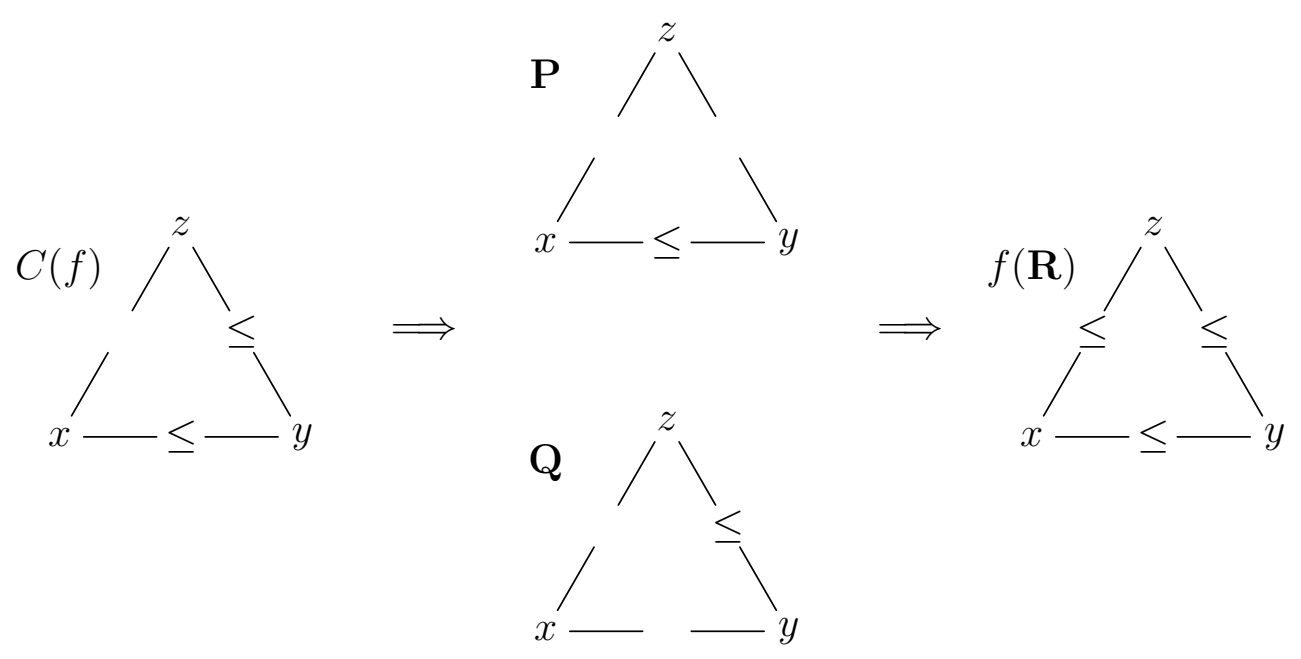

Figure 1: Transitivity of $C(f)$.

Note that since $C(f)$ is a weak preference, lemma 3.10 implies that any SWF that satisfies IIA is clerical, with cleric $C(f)$. We now define $I_{f}(x)$ to be the equivalence class of $x$ under $C(f)$. By this definition, if $y$ is in $I_{f}(x)$, then for some configurations $x \leq_{f} y$, and for some $y \leq_{f} x$. Alternatively, there is no fixed strict preference between $x$ and $y$.

Wilson's result is that $f$, restricted to $I_{f}(x)$ of size $>2$ is either null or dictatorial. We now assign each social state $x$ a dictator $D(f, x)$ as follows. If $f$ restricted to $I_{f}(x)$ is dictatorial, then we let $D(f, x)$ be that dictator. Otherwise we let $D(f, x)=0$ be the null dictator.

The following definition is the key to understanding the structure of SWFs that satisfy IIA:

Definition 3.11. Given an $S W F f$ and social state $x$, we define a series of $N+1 S W F s, f_{0}^{x}$ to $f_{N}^{x}$, recursively:

- The basis of the recursion is trivial:

$$
f_{0}^{x}:=f, \quad V_{0}:=V, \quad S_{0}:=S
$$

- For $i \geq 0$ :

Denote $w=D\left(f_{i}, x\right)$ and $A=I_{f_{i}^{x}}(x)$.

$-S_{i+1}:=A$.

- If $w=0$ then $V_{i+1}:=V_{i}$ and $f_{i+1}^{x}:=\left.f_{i}^{x}\right|_{A}$.

- If $w \neq 0$ then $V_{i+1}:=V_{i} \backslash\{w\}$ and $f_{i+1}^{x}$ is the function that $\left.f_{i}^{x}\right|_{A}$ defers to over $w$.

Lemma 3.12. When $f$ satisfies IIA then $f_{i}^{x}$ is well defined and satisfies $I I A$ for $0 \leq i \leq N$.

Proof. Clearly $f_{0}^{x}$ is well defined and satisfies IIA when $f$ does. Assume that $f_{i}^{x}$ is well defined and satisfies IIA. We will show that (1) $f_{i+1}^{x}$ is well defined and (2) satisfies IIA.

1. Since $f_{i}^{x}$ satisfies IIA, then $f_{i}^{x}$ 's preferences on the part of its domain that it doesn't share with $f_{i+1}^{x}, S_{i} \backslash S_{i+1}$, do not influence $f_{i}^{x}\left(\mathbf{R}_{i}\right)$ for states in $S_{i+1}$, and $f_{i+1}^{x}$ is well defined.

2. Since $f_{i+1}^{x}$ equals to $f_{i}^{x}$ on a restricted domain, it too satisfies IIA. 
To better understand the functions $f_{i}^{x}$, note that

1. $S_{i+1} \subseteq S_{i}$.

2. $V_{i+1} \subseteq V_{i}$ and $\left|V_{i}\right|-1 \leq\left|V_{i+1}\right| \leq\left|V_{i}\right|$.

3. If $y$ is an element of $I_{f_{i}^{x}}$ then $f_{i+1}^{y}=f_{i+1}^{x}$. Alternatively, if $x$ and $y$ are in $S_{i}$, then $f_{i}^{x}=f_{i}^{y}$.

Noting that if $y \in S_{i}$ then $f_{i}^{x}=f_{i}^{y}$, we may state the following immediate lemma:

Lemma 3.13. If $f_{i}^{x}$, restricted to some equivalence class $I_{f_{i}^{x}}(y)$, is dictatorial, then it defers to $f_{i+1}^{y}$.

Some insight may be gained by an "algorithmic" view of how $f$ "decides" on a pair $\{x, y\}$. Starting with $i=0$, this is performed as outlined in Algorithm 1.

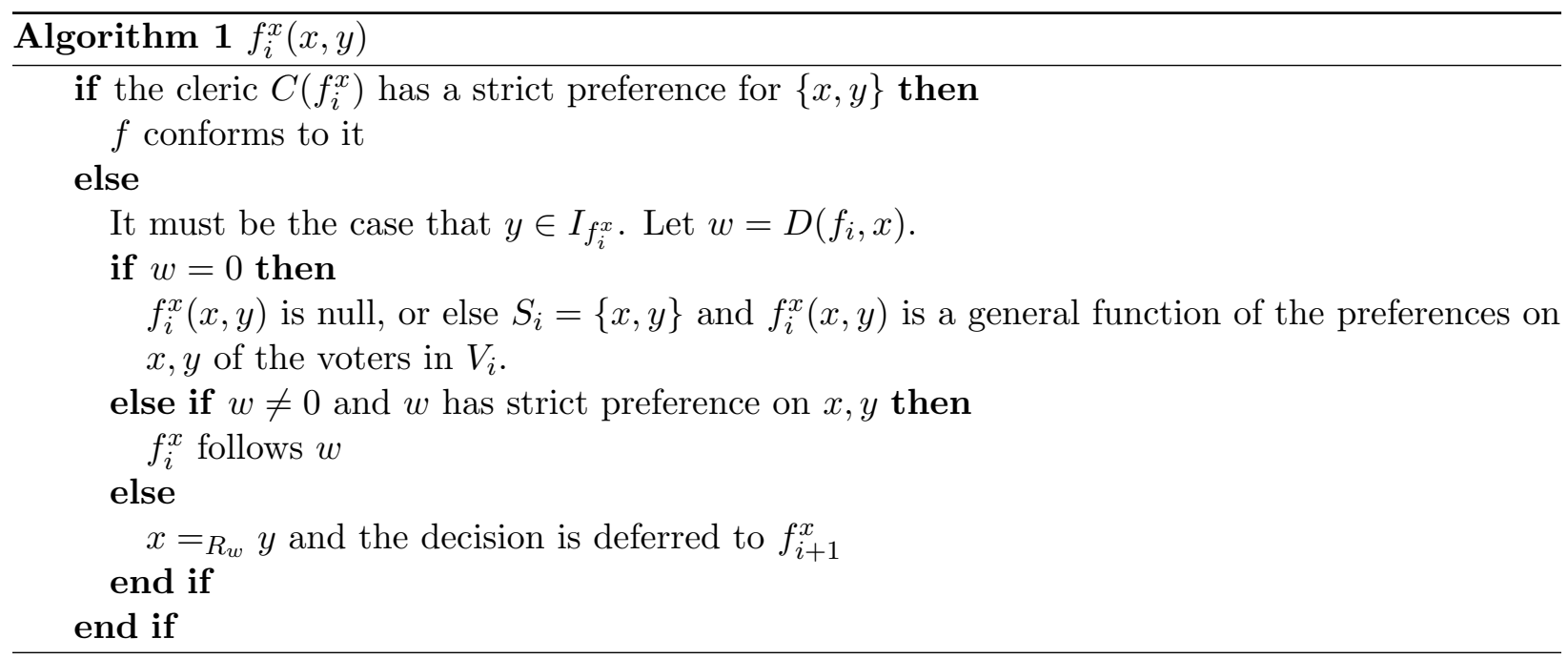

Lemma 3.14. $f_{N}^{x}$ is null or has at most two social states.

Proof. Note that if for some $i$ we have $V_{i+1}=V_{i}$ then $f_{N}^{x}=f_{i}$ has at most two social states or is null. Since $V_{i+1}$ can be smaller than $V_{i}$ for no more than $N$ values of $i$, the proof follows.

Therefore trivially $f_{N}^{x}$ is by definition clerical-dictatorial. We may now prove theorem 3.9: any SWF which satisfies IIA is clerical-dictatorial.

Proof. Let $f$ satisfy IIA. Then the function $f_{i}^{x}$ is well defined for all $x \in S$ and $0 \leq i \leq N$ by lemma 3.12. Furthermore, it satisfies IIA.

Now, for all $x, f_{N}^{x}$ is clerical-dictatorial by lemma 3.14. Assume that $f_{i+1}^{x}$ is clerical dictatorial for all $x$. We will show that $f_{i}^{x}$ is clerical-dictatorial, and thus prove by induction that $f=f_{0}^{x}$ is too.

If $f_{i}^{x}$ is null or has only one or two social states then it is clerical dictatorial. Otherwise, it is clerical with cleric $C\left(f_{i}^{x}\right)$. Let $A=I_{f_{i}^{x}}(y)$ be some equivalence class of $C\left(f_{i}^{x}\right)$ of size $>2$. Then

1. Since $f_{i}^{x}$ satisfies IIA then $\left.f_{i}^{x}\right|_{A}$ is well defined. As a consequence of the same fact, by theorem 3.3 (Wilson), $\left.f_{i}^{x}\right|_{A}$ is either dictatorial, null, or of size 2. 
2. If $\left.f_{i}^{x}\right|_{A}$ is dictatorial then it defers to $f_{i+1}^{y}$ by lemma 3.13. By the inductive hypothesis $f_{i+1}^{y}$ is clerical-dictatorial.

Thus $f_{i}^{x}$ satisfies the conditions for being clerical-dictatorial.

\subsection{Clerical Dictatorial $\Longrightarrow$ IIA}

Theorem 3.15. A clerical-dictatorial SWF satisfies IIA.

Proof. The proof is by induction on the number of voters. The base case of no voters is trivial. Now assume that there are $n \geq 1$ voters and let $f: \Pi_{S}^{V} \rightarrow \Pi_{S}$ be a clerical-dictatorial SWF, with cleric $C$. Let $\mathbf{P}$ and $\mathbf{Q}$ be configurations that agree on $\{x, y\}$. We would like to show that $f(\mathbf{P})$ agrees with $f(\mathbf{Q})$ on $\{x, y\}$.

If $C(f)$ is strict about $\{x, y\}$ then $f$ is constant with regard to them, so in particular $f(\mathbf{P})$ agrees with $f(\mathbf{Q})$ on $\{x, y\}$. Otherwise, $y \in I_{f}(x)$, and we denote $A=I_{f}(x)$. By the definition of clerical-dictatorial functions, $\left.f\right|_{A}$ is well defined, so that the ranking of $x$ and $y$ depends only on the voters' opinions with regards to states in $A$.

The case where $\left.f\right|_{A}$ is null is trivial. Likewise, if $I_{f}(x)$ is of size two then we're done as the ranking between $x$ and $y$ depends only on the individual preferences between them.

In the remaining case $\left.f\right|_{A}$ is dictatorial. Let $i$ be the dictator and $g$ the clerical-dictatorial function that it defers to. Now if the dictator $i$ has strict ranking between $x$ and $y$ in $P_{i}$ and $Q_{i}$ then $f(\mathbf{P})$ and $f(\mathbf{Q})$ follow the dictator and agree on $\{x, y\}$. Otherwise, the relative ranking of $x$ and $y$ is determined by $g$, which is IIA by the inductive hypothesis, and so $f(\mathbf{P})$ and $f(\mathbf{Q})$ agree on $\{x, y\}$ as needed.

\subsection{Arrow's Model and Wilson's Model}

The following two theorems, also mentioned above, are simple applications of our main result to the domains of Arrow's and Wilson's models, i.e. the set of SWFs satisfying, besides IIA, WPP and CS, respectively.

Our "if and only if" version of Arrow's theorem is:

Theorem (3.6). An SWF satisfies IIA and WPP if and only if is dictatorial (with the dictator's decrees followed verbatim rather than in reverse), and defers to a clerical-dictatorial function.

Proof. First direction: an SWF which satisfies IIA and WPP must be dictatorial (with the dictator's decrees followed verbatim rather than in reverse), by Arrow's theorem. By our theorem, it must be clerical-dictatorial and hence defer to a clerical dictatorial function.

Second direction: an SWF which is dictatorial (again, verbatim) certainly satisfies WPP. If it is also clerical-dictatorial, then it also satisfies IIA.

Our "if and only if" version of Wilson's first theorem is:

Theorem (3.7). An SWF satisfies IIA and CS if and only if is null or dictatorial, and in the latter case defers to a clerical-dictatorial function.

The proof of this theorem is essentially identical to the previous. 
Proof. First direction: an SWF which satisfies IIA and CS must be null or dictatorial, by Wilson's theorem. By our theorem, it must be clerical-dictatorial and hence defer to a clerical dictatorial function.

Second direction: an SWF which is dictatorial or null certainly satisfies CS. If it is also clericaldictatorial, then it also satisfies IIA.

\section{The Number of Social Welfare Functions Satisfying IIA}

The characterization of (transitive) social welfare functions satisfying IIA as clerical-dictatorial facilitates counting the number of such functions, for low numbers of social states.

The sequence starting with $1,1,13,75,541, \ldots$, which counts the number of clerics (weak preferences) over $S$ social states, is known as the Ordered Bell Numbers or Fubini Numbers [8]. There is no known simple expression for this sequence, recursive or otherwise.

Denote by $q_{s}(v)$ the number of SWFs satisfying IIA, over $s$ social states and $v$ voters. Then $q_{s}(0)$ are the Ordered Bell Numbers. For small $s$, it is possible to form a recursive expression for $q_{s}(v)$. We do that by first choosing a cleric $C$, and then counting the number of functions for each equivalence class.

For $s=1$, only one function is possible. For $s=2$, any function is transitive and satisfies IIA, and therefore

$$
q_{2}(v)=3^{3^{v}}
$$

For $s=3$

$$
q_{3}(v)=6+6 \cdot\left(q_{2}(v)-2\right)+\left(1+2 v q_{3}(v-1)\right)
$$

with $q_{3}(0)=13$. The first addend counts the number of strict clerics, that is, the number of fixed strict functions. There are six such clerics, and each equivalence class is of size one and therefore has only one possible function.

The second addend counts the number of functions for which $C$ has an equivalence class of size one and an equivalence class of size two. There are six such clerics, and for each one there are $q_{2}(v)$ possible functions for the size two equivalence class. Of these, we have to subtract the two constant strict ones which we counted in the third addend.

The third addend counts the number of functions possible when $C$ is the unique cleric that deems all three social states equal. Then, either the function is constant and equal to $C$, or else it is dictatorial. There are $v$ possible dictators, and each one can be followed directly or inversely. Once that is chosen, there are $q_{3}(v-1)$ options for the function that that dictator defers to.

Note that $q_{3}(v)$ is dominated by the second addend, or by the number of functions over two social states.

A similar analysis, although increasingly complicated, can be done for larger $s$. For example, for $s=4$,

$$
q_{4}(v)=24+36 \cdot\left(q_{2}(v)-2\right)+6 \cdot\left(q_{2}(v)-2\right)^{2}+8 \cdot\left(1+2 v q_{3}(v-1)\right)+\left(2 v q_{4}(v-1)+1\right) .
$$

Here, $q_{4}(v)$ is dominated by the third addend, or the number of functions for which $C$ has two equivalence classes of size two.

\subsection{Arrow's Model}

The calculation of the number of SWFs satisfying Arrow's conditions is simple, when expressed in terms of $q_{s}(v)$. Let $r_{s}(v)$ be the number of SWFs satisfying IIA and the Weak Pareto Principle. Then $r_{s}(0)$ is equal to $q_{s}(0)$, or the Ordered Bell Numbers. 
For $v>1$ and $s>1$ :

$$
r_{s}(v)=v q_{s}(v-1) .
$$

Since we must pick a dictator and the clerical-dictatorial function that is deferred to.

\subsection{Wilson's Model}

The calculation in this case is also simple, when expressed in terms of the $q_{s}(v)$. Let $p_{s}(v)$ be the number of SWFs satisfying IIA and Citizens' Sovereignty. Then $p_{s}(0)$ is one, since the only possible function is the null function.

For $s=2$, any function is admissible, except the two that have constant strict rankings:

$$
p_{2}(v)=3^{3^{v}}-2 .
$$

For $s=3$

$$
p_{3}(v)=1+2 v q_{3}(v-1)
$$

which is the last term in the expression for $q_{3}(v)$, the term which counts the number of functions in the case where $C(f)$ deems all alternatives equal. As mentioned above, this conditions is equivalent to Citizens' Sovereignty. Likewise, for any $s>2$ :

$$
p_{s}(v)=1+2 v q_{s}(v-1) .
$$

\section{References}

[1] K. Arrow. A difficulty in the concept of social welfare. J. of Political Economy, 58:328-346, 1950 .

[2] K. Arrow. Social choice and individual values. John Wiley and Sons, 1963.

[3] J. Blau. Arrow's theorem with weak independence. Econometrica, 538(152):413-420, 1971.

[4] J. Buchanan. Individual choice in voting and the market. The Journal of Political Economy, 62(4):334-343, 1954.

[5] J. Buchanan. Social choice, democracy, and free markets. The Journal of Political Economy, 62(2):114-123, 1954.

[6] G. Kalai. A Fourier-theoretic perspective on the Concordet paradox and Arrow's theorem. Adv. in Appl. Math., 29(3):412-426, 2002.

[7] E. Mossel. A quantitative arrow theorem. Available at the Arxiv 0903.2574, 2009.

[8] Wikipedia. Strict weak ordering - Wikipedia, the free encyclopedia, 2009. [Online; accessed 15-July-2009].

[9] R. Wilson. Social choice theory without the pareto principle. Journal of Economic Theory, 5(3):478-486, December 1972. 